\title{
Robust Trajectory Tracking and Visual Servoing Schemes for MEMS Manipulation
}

\author{
Brahim Tamadazte, Nadine Le-Fort Piat, and Sounkalo Dembélé
}

\begin{abstract}
This paper focuses on the automation of manipulation and assembly of microcomponents using visual feedback controls. Trajectory planning and tracking methods are proposed in order to avoid occlusions during microparts manipulation and to increase the success rate of pick-and-place manipulation cycles. The methods proposed are validated using a five degree-of-freedom (DOF) microrobotic cell including a 3 DOF mobile platform, a 2 DOF micromanipulator, a gripping system and a top-view imaging system. Promising results on accuracy and repeatability of microballs manipulation tasks are obtained and presented.
\end{abstract}

\section{INTRODUCTION}

The development of micro electromechanical systems (MEMS) as well as micro opto electromechanical systems (MOEMS) leads to smaller components and increasingly complex microstructures thus requiring sophisticated micromanipulation techniques. Furthermore, no commercial success of these products is possible without reliable and costeffective assembly and packaging technologies. Therefore, research conducted in the past two decades has led to the development of microrobotic manipulation and assembly setup [3], [15], [8], [4]. Meanwhile, the availability of high resolution cameras and powerful microprocessors has enabled vision systems to play a key role in the automation of robotic microassembly stations. Several vision techniques combined with control ones have been successfully developed and implemented. The vision-based feedback control techniques applied to the microdomain include image-based visual servoing [9], multiscale visual servoing [11], [13], pose-based visual servoing [12] and hybrid force/vision control [2].

Image-based visual control (IBVS) is more frequently used in MEMS manipulation. It has shown its relevance and high level of precision. But some drawbacks remain notably the low level of robustness due to the fact that trajectories of the microparts are not checked during the positioning and orientation tasks. This often leads either to occlusions of the microparts by the gripper or to the exit of the parts outside the field-of-view of the microscope and then to failures of the manipulation tasks.

This paper investigates trajectory tracking using visionbased control law in order to prevent the passage of micropart under the gripping system or it exit outside of the microscope field-of-view. The trajectory to track is obtained using a basic approach based on potential fields (i.e. repulsive and attractive). Firstly, this technique allows a first coarse estimation of

Brahim Tamadazte, Nadine Le-Fort Piat and Sounkalo Dembélé are with FEMTO-ST Institute-AS2M/UMR CNRS 6174 /UFC/ ENSMM / UTBM, 24 rue Alain Savary, 25000 Besançon, France, brahim.tamadaztedens $2 \mathrm{~m}$. fr the corresponding points of the micropart to reach the desired position between the fingers of the gripper (positioning task). A piecewise Bézier interpolation is after applied to obtain a trajectory with properties of tension and continuity. Secondly, an approach coupling the trajectory tracking and an imagebased control law is applied to resolve the problem of total or partial occlusions of the microparts.

The paper is organized as follows: in section II, the microassembly workcell based on high precision positioning systems, an imaging system and a gripping system is presented. Section III describes the trajectory planning; the real-time trajectory tracking and the visual-based control law associated enabling more precise and robust automated manipulations. Section V gives experimental results on microballs manipulation.

\section{MiCROASSEMBLY WORKCELL}

The experimental set-up used to validate the concepts developed includes a robotic system in combination with a gripping system and an imaging system. The whole set-up is positioned on a vibration-free table inside a controlled environment as required by this kind of experiment (Fig. 1).

Two PCs connected by an Ethernet link process the information: the first (Pentium (R) D, CPU $2.80 \mathrm{G} \mathrm{Hz}, 2$ Go of RAM) is dedicated to vision algorithms while the second (Pentium(R) 4, CPU 3.00 G Hz, and 1 Go of RAM) is used for control algorithms. From a kinematic point of view the workstation is a five DOF robotic system. Three DOF in translation are achieved by three high accuracy linear stages and two DOF in rotation are achieved by two high accuracy angular stages (all from Polytec PI). The five DOF are distributed into two robotic systems: a $x y \theta$ system and a $\mathrm{z} \varphi$ system. The former system (the positioning platform) is equipped with a compliant table (the table is supported by three springs) and enables the positioning in the horizontal plane of microparts (Fig. 1.(b)). The latter system (the manipulator) supports the gripper and enables the vertical positioning and spatial orientation of microparts. The gripper actuated by piezo-electric bimorphs can grab a wide diversity of micro-objects (Fig. 1.(c)). The imaging system includes three videomicroscopes, one positioned vertically and the two others positioned laterally. Only the vertical one is used in the present experiments. It is an optical videostereomicroscope of the type LEICA MZ 16 A. The zoom (and then the magnification) and the focus are motorized and controlled by the vision PC. The magnification ranges from $0.71 \times$ to $11.5 \times$. The field-of-view varies from $700 \mu \mathrm{m} \times$ $900 \mu \mathrm{m}$ with a resolution of $1.4 \mu \mathrm{m}$ at the maximum of the 


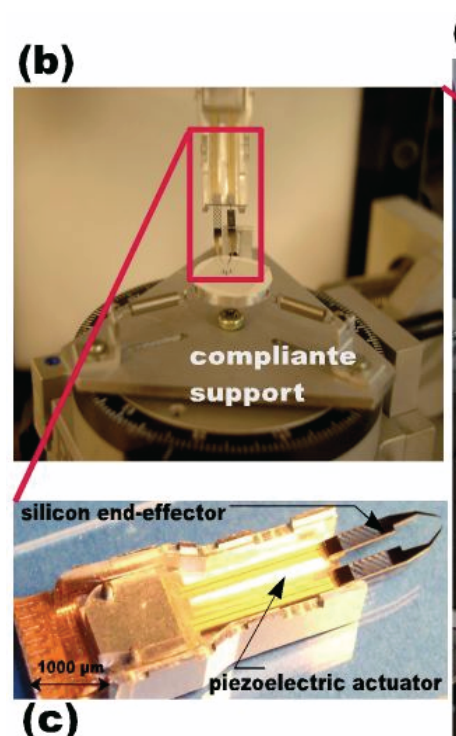

(a)

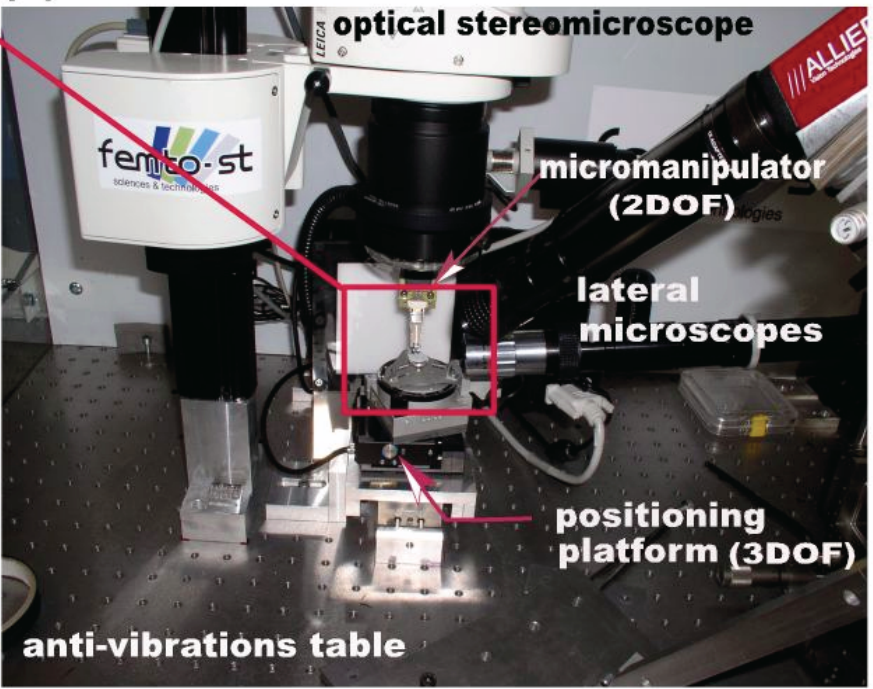

Fig. 1. Shots of the 5 DOF microassembly workcell. (a) showing the global view, (b) representes a zoom on the 3 DOF positioning platform and the 2 DOF micromanipulator, and (c) illustrates the 4 DOF gripping system.

magnification $(11.5 \times)$ to $20 \mathrm{~mm} \times 25 \mathrm{~mm}$ with a resolution of $21 \mu \mathrm{m}$ at the minimum of magnification $(0.71 \times)$. The depth-of-field varies from $2.9 \mathrm{~mm}$ to $0.035 \mathrm{~mm}$ according to the numerical aperture of the objective. The work distance is approximately $112 \mathrm{~mm}$.

\section{MEMS MANIPULATION}

In previous works [13], we have performed pick-andplace cycles of silicon square micro-objects by means of visual feedback controls. The success rate was about $72 \%$ despite the high precision of these controls. The following causes of failure were demonstrated: adhesion forces (the micropart remained stuck to the gripper tips during the release task), electrostatic forces (the micropart was projected during the reaching of the gripper to the micropart) and trajectory of the micropart (the micropart was hidden by the gripper or moved outside the microscope field-of-view during the orientation and positioning tasks (Fig. 2). This section investigates the latter problem and makes the control more robust by implementing a trajectory planning associated to the visual control.

\section{A. Path Planning}

Among the path planning methods existing in the field of mobile robotics [7], [6], the potential field approach is adopted in this paper thanks to its simplicity: the micropart to manipulate is considered as a particle in the configuration space Q. Let the micropart to pick-and-place be a $200 \mu \mathrm{m}$ diameter metal ball. This particle is subjected to the artificial potential field $U(q(x, y, t))$ where $q(x, y, t)$ is the position of the micropart at time $t$ (Fig. 2). The direction of the microball induced by the artificial force $F(q(x, y, t))$ can be represented by:

$$
F(q(x, y, t))=-\nabla U(q(x, y, t))
$$

The field $U(q(x, y, t))$ is defined as the sum of an attractive potential field $U_{\text {att }}$ pushing the microball to the final position (desired position) and a repulsive term $U_{\text {rep }}$ taking the ball away from the gripping system (the edge of the gripper in the image) and the image borders.

$$
U(q)=U_{\text {att }}(q)+U_{\text {rep }}(q)
$$

The attractive field can be defined simply as a parabolic of the following equation:

$$
U_{\text {att }}(q)=\frac{1}{2} \xi \rho_{\text {goal }}^{2}(q)
$$

with $\xi$ a positive scalar, $\rho_{\text {goal }}$ the euclidean distance $\left\|q-q_{\text {goal }}\right\|$ ( $q_{\text {goal }}$ i.e. the desired position).

The repulsive potential is used to create potential barriers around the obstacles which correspond in the present case to some selected pixels belonging to the gripper edges or to the image borders (Fig. 2). It can be defined by:

$$
\begin{gathered}
U_{\text {rep }}(q)=\frac{1}{2} \eta\left(\frac{1}{\rho_{o b s}(q)}-\frac{1}{\rho_{0}}\right)^{2} \quad \text { if } \quad \rho_{o b s}(q)<\rho_{0} \\
U_{\text {rep }}(q)=0 \quad \text { if } \quad \rho_{o b s}(q) \geqslant p_{0}
\end{gathered}
$$

where:

- $\eta$ is a positive scalar,

- $\rho_{0}$ is a positive scalar called influence distance of the pixelobstacle,

- $\rho_{o b s}$ is the euclidean distance $\left\|q-q_{o b s}\right\|$ ( $q_{o b s}$ is the position of the obstacle). 


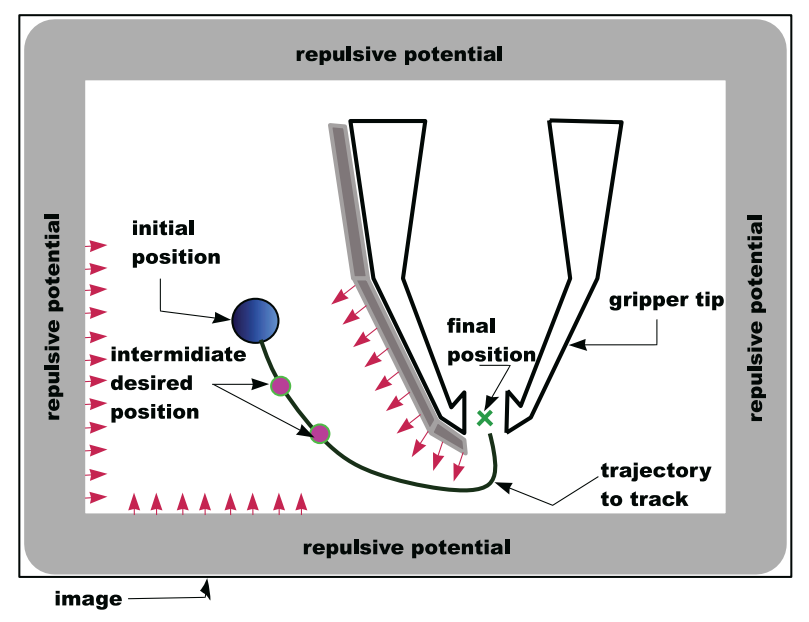

Fig. 2. Illustration of the approach.

The function $U_{\text {rep }}$ is positive or null and tends to $\infty$ when the micropart reaches the pixel-obstacle border.

Once the potential fields are identified, the gradient descent method is used to compute (roughly) the crossingpoints of the ball to avoid occlusions by the obstacles. The gradient descent consists in following the direction of the force $F(q(x, y, t))$ and forwarding in this direction with the step $\Delta_{i}$.

$$
\begin{aligned}
& x\left(q_{i+1}\right)=x\left(q_{i}\right)+\Delta_{i} \frac{\partial U}{\partial x(x, y)} \\
& y\left(q_{i+1}\right)=y\left(q_{i}\right)+\Delta_{i} \frac{\partial U}{\partial y(x, y)}
\end{aligned}
$$

\section{B. Trajectory Parameterization using Bézier Curves}

When the corresponding points are computed using the path planning proposed previously, Bézier curves are implemented in order to interpolate and to parameterize the curve (trajectory) between the initial and final position of the microball. The simplest method to estimate Bézier curves is to evaluate them at several closely spaced points (called corresponding points in this paper), to scan and to convert the approximating sequence of line segments.

$$
F(\sigma)=\sum_{k=0}^{3} m_{k} \frac{3 !}{k !(3-k)} \sigma^{k}(1-\sigma)^{3-k}
$$

After simple developments, we obtain:

$$
\begin{aligned}
F(\sigma) & =\sigma^{3}\left(m_{3}+3\left(m_{1}+m_{2}\right)-m_{0}\right)+3 \sigma^{2}\left(m_{0}-2 m_{1}+m_{2} \backslash 9\right) \\
& +3 \sigma\left(m_{1}-m_{0}\right)+m_{0}
\end{aligned}
$$

where $m_{3}, m_{2}, m_{1}$ and $m_{0}$ are the control points for a given curve and $\sigma$ represents the parameterization used to produce the function $F(\sigma)$ and the parameter $\sigma \in[0,1]$.

\section{Robust Visual Tracking Algorithm}

There are several methods of visual tracking which are usually classified into two groups. The first group consists of the tracking of local features like lines, segments, points, edges, etc. [14]. The results of these techniques highly depend on the quality of the images and remain very sensitive to feature detection [5]. The other group contains methods that perform a comparison between two frames in the image sequence by minimizing an error based on the image brightness. These methods take into account some parameters like motion, deformation or illumination parameters between the two frames or the frame and a template.

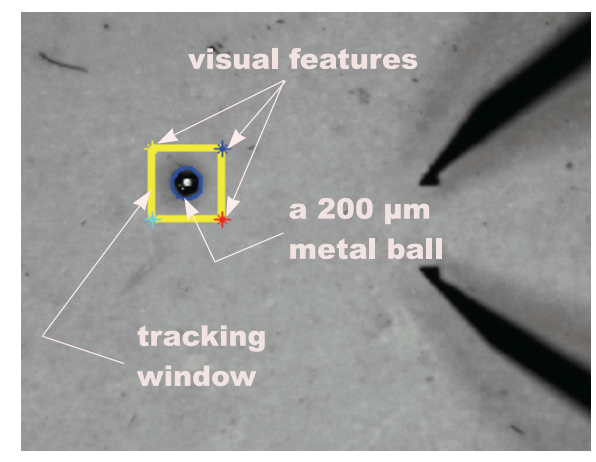

Fig. 3. Image captured during the microball tracking.

Images of the planar silicon microparts as well as those of the gripper tips are not very sharp because of the properties of the microscope. Consequently, a robust tracking algorithm able to work in real-time is required. The tracking is achieved by an algorithm proposed in [5] and [10]. This algorithm is based on the second-order-minimization. It has a far superior convergence rate than other techniques, which is an important property for real-time tracking. For instance, if we consider the window $I^{*}$ of $m$ pixels which contains the micro-object as the reference, then tracking $I^{*}$ in the global current image $I$ consists in computing a vector $V$. This vector of $(8 \times 1)$ size includes the parameters of the projective transformation $H$. It transforms each pixel $p^{*}$ from the reference image $I^{*}$ to the current image $I$ using the following relationship:

$$
I\left(w(H)\left(p_{i}^{*}\right)\right)=I^{*}\left(p_{i}^{*}\right)
$$

In [1], for an approximation $\hat{V}$ of $V$, or $\hat{H}$ of $H$, the problem is to find an incremental transformation $H(V)$ such as the difference between the region image $I$ transformed with the composition $w(\hat{H}) \circ w(H(V))$, and the corresponding region in the image $I^{*}$ is equal to zero. It involves to find the vector $V$ such that $\forall i \in(1,2, \ldots, q)$, we have:

$$
y_{i}(V)=I\left(w(\hat{H}) \circ w(H(V))\left(p_{i}^{*}\right)\right)-I^{*}\left(p_{i}^{*}\right)=0
$$

where $w(H)$ represents an automorphism transformation.

Let be the $(\mathrm{q} \times 1)$ vector $y(V)$ which represents these differences between $I$ and $I^{*}$. Therefore, we can write:

$$
y_{i}(V)=\left[y_{1}(V), y_{1}(V), \cdots y_{q}(V)\right]^{\top}
$$


The solution of the equation (13) can be computed by finding $V=\tilde{V}$ which verifies the following system:

$$
y(\tilde{V})=0
$$

\section{Visual Control Law}

From a general point of view, the aim of vision-based control schemes is to minimize the error e $(\mathrm{t})$ typically defined by:

$$
\mathbf{e}(t)=\mathbf{s}(\mathbf{m}(t))-\mathbf{s}^{*}
$$

where $\mathbf{m}$ is a set of visual information $\mathbf{s}$ extracted from the image (e.g. the image coordinates of interest points) and $\mathbf{s}^{*}$ the desired visual features. In our case, the vector $\mathbf{m}$ contains the coordinates of the four corners of the bonding box (tracking window) delimiting the micro-object to be handling. It can be noted that the coordinates of the desired position $\mathbf{s}^{*}$ is function of the time $t$. Therefore, e can be defined by:

$$
\mathbf{e}(t)=\mathbf{C}\left(\mathbf{s}(t)-\mathbf{s}^{*}(t)\right)
$$

where $\mathbf{C}$ represents the $6 \times k$ combination matrix and $k$ the number of the visual information $\mathbf{s}$.

Let the spatial velocity of the camera (optical microscope) be denoted by:

$$
\mathbf{v}=(v, \omega)
$$

where $\mathbf{v}$ is the instantaneous linear velocity and $\omega$ is the instantaneous angular velocity. In our case, 3 DOF micropositioning platform (translations $x y$ and rotation $\theta$ ) are considered, then

$$
\begin{aligned}
v & =\left(v_{x}, v_{y}\right)^{\top} \\
\omega & =\omega_{\theta}
\end{aligned}
$$

To track the image trajectory using an image-based visual servoing control scheme, it can be used to following vision-based task function e to be regulate to zero:

$$
\mathbf{e}=\mathbf{L}_{\mathbf{s}}^{+}\left(\mathbf{s}(t)-\mathbf{s}^{*}(t)\right)
$$

with $\mathbf{L}_{\mathbf{s}}^{+}$is the pseudo-inverse of the interaction matrix $\mathbf{L}_{\mathbf{s}}$ associated to the visual information $\mathbf{s}$. The exponential decay of e toward zero can be obtained by imposing:

$$
\dot{\mathbf{e}}=\lambda \mathbf{e}
$$

where $\lambda$ being a proportional positive gain.

The corresponding control law is given by:

$$
\mathbf{v}=\mathbf{W}^{+}{ }_{1}+\mathbf{W}^{+}{ }_{2}
$$

with

$$
\mathbf{W}^{+}{ }_{1}=-\lambda \widehat{\mathbf{L}_{\mathbf{s}}^{+}}\left(\mathbf{s}(r, t)-\mathbf{s}^{*}(t)\right)
$$

represents the part of the interaction matrix which regulating to zero the error between $\mathbf{s}$ and $\mathbf{s}^{*}$ at the time $t$, and

$$
\mathbf{W}_{2}^{+}=\widehat{\mathbf{L}_{\mathbf{s}}^{+}} \frac{\partial \mathbf{s}^{*}(t)}{\partial t}
$$

allows to compensate the tracking error where $\widehat{\mathbf{L}_{\mathbf{s}}^{+}}$is the approximation of the pseudo-inverse of the interaction matrix $\mathbf{L}_{\mathbf{s}}$.

As mentioned above the coordinates of the four corners of the bonding box (tracking windows) delimiting the microobject are chosen to compute the interaction matrix $\mathbf{L}_{\mathbf{s}}$ given by:

$$
\begin{aligned}
& \mathbf{L}_{\mathbf{s}}(x(k), z)=\left(\begin{array}{cccccc}
\frac{-1}{z} & 0 & \frac{x_{1}}{z} & x_{1} y_{1} & -1-x_{1}^{2} & y_{1} \\
\frac{-1}{z} & 0 & \frac{x_{2}}{z} & x_{2} y_{2} & -1-x_{2}^{2} & y_{2} \\
\frac{-1}{z} & 0 & \frac{x_{3}}{z} & x_{3} y_{3} & -1-x_{3}^{2} & y_{3} \\
\frac{-1}{z} & 0 & \frac{x_{4}}{z} & x_{4} y_{4} & -1-x_{4}^{2} & y_{4}
\end{array}\right) \\
& \mathbf{L}_{\mathbf{s}}(y(k), z)=\left(\begin{array}{cccccc}
0 & \frac{-1}{z} & \frac{y_{1}}{z} & 1+y_{1}^{2} & -x_{1} y_{1} & -x_{1} \\
0 & \frac{-1}{z} & \frac{y_{2}}{z} & 1+y_{2}^{2} & -x_{2} y_{2} & -x_{2} \\
0 & \frac{-1}{z} & \frac{y_{3}}{z} & 1+y_{3}^{2} & -x_{3} y_{3} & -x_{3} \\
0 & \frac{-1}{z} & \frac{y_{4}}{z} & 1+y_{4}^{2} & -x_{4} y_{4} & -x_{4}
\end{array}\right)
\end{aligned}
$$

\section{EXPERIMENTAL RESULTS}

The concepts described above have been validated using the 5 DOF microassembly workcell presented in section II. The task studied consists of the automatic positioning of microparts between the gripper end-effectors. These microparts are $200 \mu \mathrm{m}$ diameter metal microballs of bearings of watches. The microballs are randomly placed on the positioning platform using a feeding system. So, the initial positions of the microballs change during the different pick-and-place cycles. Therefore, it is necessary to plan the trajectory of the microparts during the automatic pick-and-place processes. In this case, only the 3 planar DOF on the microassembly workcell is used. Once the micropart is perfectly positioned (Fig. 5), the micromanipulator is controlled is order to descent the gripper to grasp the micropart (Fig. 7.(a)). The manipulator and the gripper closing tasks are also performed in an automatic way using a 2D visual approach (for more information, refer [13]). Once the microballs are grasped then they are moving in order to be inserted in their respective targets (Fig. 7.(c)).

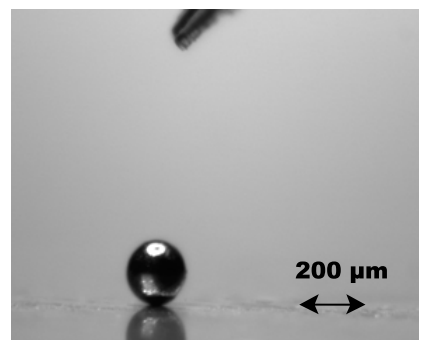

Fig. 5. Side view on the microball positioned under the gripper.

Figure 4 shows the evolution in the time of the positioning error along $x$ and $y$. Each curve presents three parts. The 


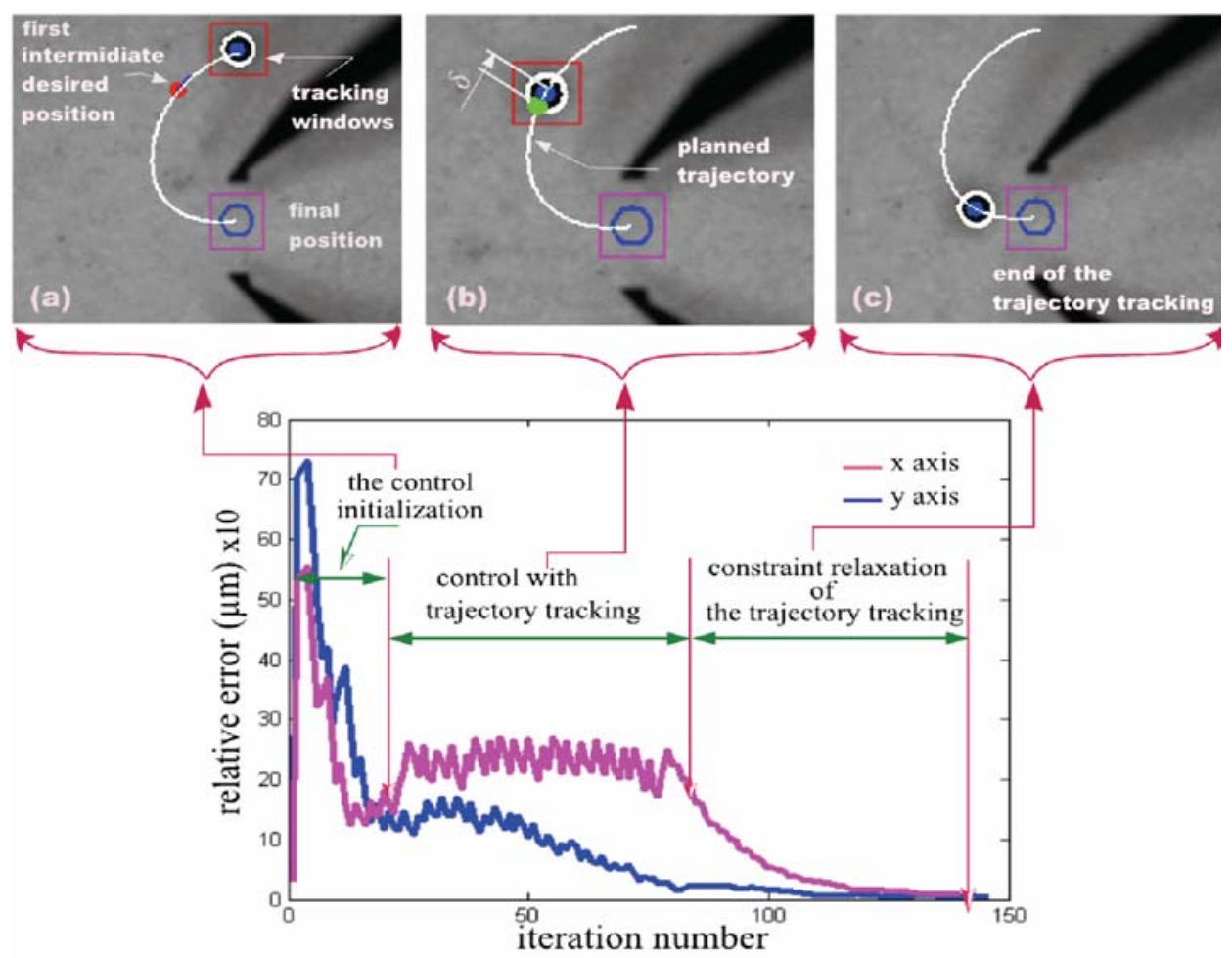

Fig. 4. Relative errors during the positioning of the $200 \mu \mathrm{m}$ diameter metal ball using the image-based visual servoing in association with the trajectory tracking.

first part corresponds to the initialization stage of the trajectory tracking (Fig. 4.(a)), the second part represents the trajectory tracking stages (Fig. 4.(b)) and the last part shows the relaxation constraint of the trajectory tracking and the convergence of the errors to zero (Fig. 4.(c)). A very interest accuracy is obtained for the different pick-and-place cycles. A mean positioning error of $1.4 \mu \mathrm{m}$ is obtained for a series of 20 automatic positioning using the proposed approaches.

Figure 6 presents some examples of trajectories corresponding to different initial positions of the microball according to the gripper. Several experimental results of the trajectory planning and the trajectory tracking approaches are shown. Figures 6.(a), (b) and (c) illustrate an intermediate position of the metal microball (trajectory tracking steps). Figures 6.(d), (e) and (f) show the positioning task of a 200 $\mu \mathrm{m}$ microball at the initial position.

Figure 7 presents a shot showing the microball inside the fingers of the gripper. The quality of the gripping indicates the relevance of the approach: the microball is positioned with precision and can be grabbed easily (Fig. 7.(b)).

\section{CONCLUSION AND FUtURE PERSPECTIVES}

In this paper, we have focused on methods for resolving the problem of occlusions during MEMS manipulations. A method of path planning based on attractive and repulsive potential fields allows determining roughly the corresponding points. An interpolation by Bézier curves leads to a precise and parameterized trajectory from the initial position of the micro-object to the target position. Then an imagebased visual servoing is combined with the tracking of the trajectory in order to perform the positioning and the centering subtasks without risk of occlusions of the microparts. Interesting results have been obtained in automatic handling of $200 \mu \mathrm{m}$ metal microballs of a microbearing on our micromanipulation workcell. The accuracy and repeatability of micromanipulation tasks have been improved by applying the proposed methods. For instance, a mean positioning error of $1.4 \mu \mathrm{m}$ is obtained for several pick-and-place cycles performed.

The next stages of this work will concern the integration of the methods presented in this paper in a full microassembly process. This integration will allow to seriously improve the success rate of the automation of MEMS and MOEMS assembly using vision feedback control.

\section{AKNOWLEDGEMENTS}

This work is partially conducted with financial support from the project "Hybrid Ultra Precision Manufacturing Process Based on Positional and Self assembly for Complex Micro-Products (HYDROMEL NMP2-CT-2006026622)" funded by the European Commission.

The authors would like to thank Eric Mermet, $\mathrm{PhD}$ student from the COGIT Laboratory for his help in programming of the potential simulation. 


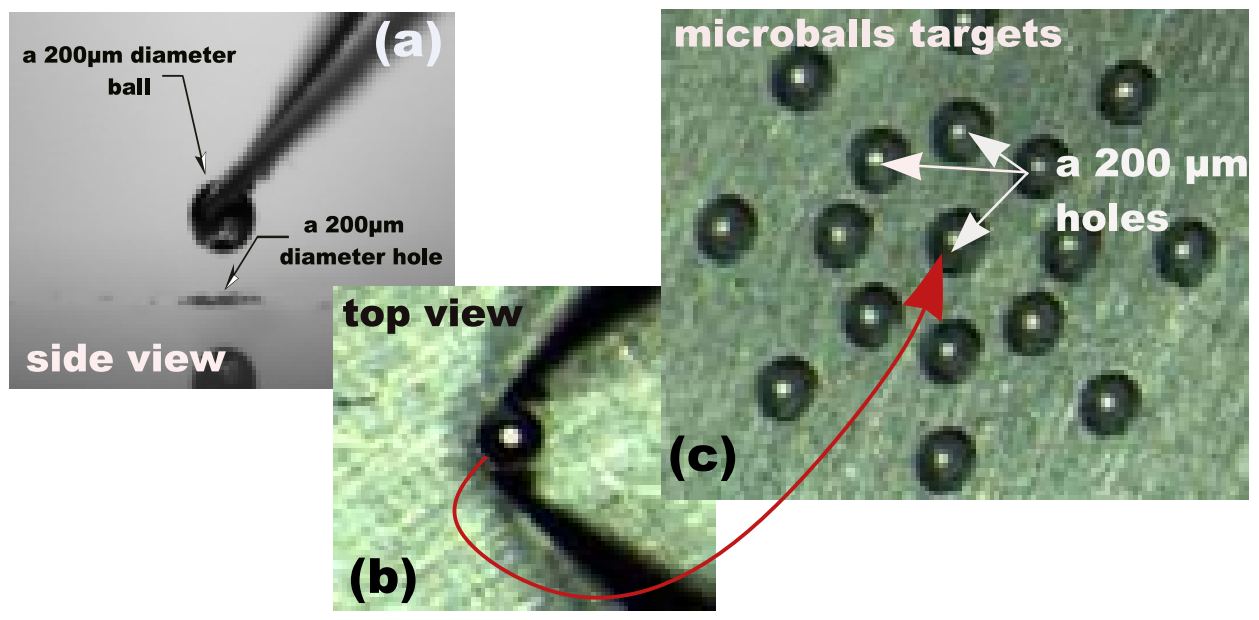

Fig. 7. Shot showing the microball inside the fingers of the gripper.

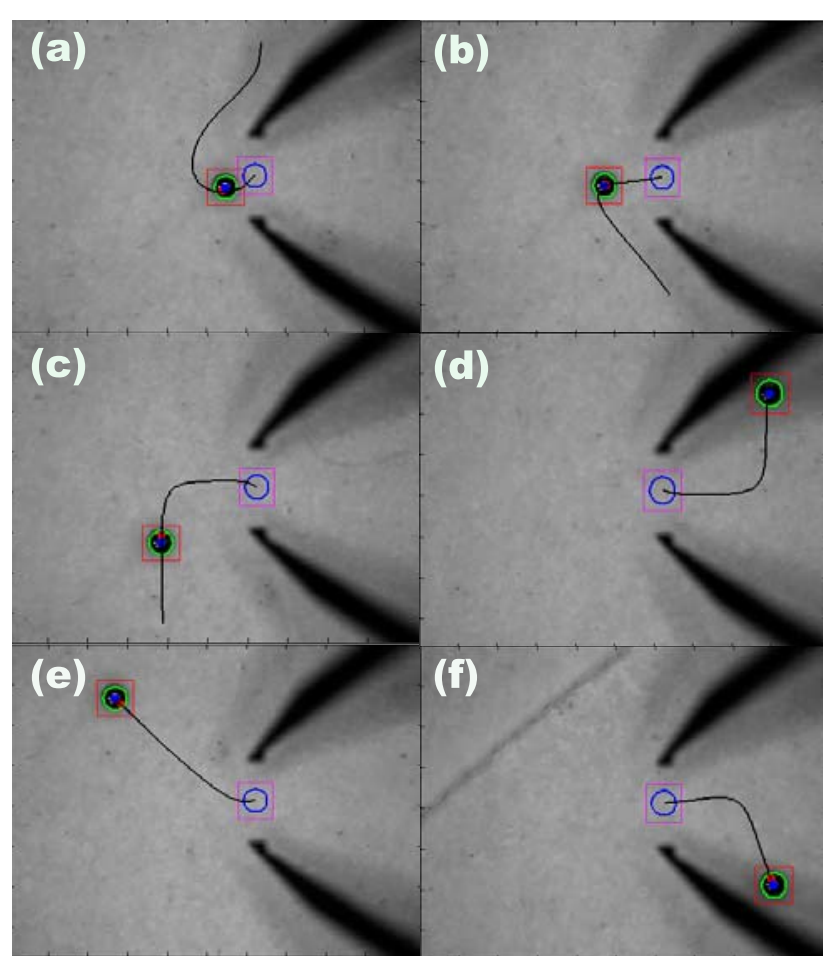

Fig. 6. Some trajectories demonstrating the avoidance of occlusion of the microball by the gripper fingers.

\section{REFERENCES}

[1] S. Benhimane and E. Malis, Homography-based 2D visual tracking and servoing, The International Journal of Robotics Research vol. 26, No. 7, 2007, pp. 661-676.

[2] L. Chen, W. Rong Sun, L. and, and X. Bian, Hybrid control of vision and force for MEMS assembly system, IEEE International Conference on Robotics and Biometrics Shenyang, China, August 2004, pp. 136141.

[3] N. Dechev, L. Ren, W. Liu, W.L. Cleghorn, and J.K. Mills, Development of a 6 degree of freedom robotic micromanipulation for use in 3D MEMS microassembly, IEEE International Conference on Robotics and Automation, Orlando, Fl., May 2006, pp. 281-288.
[4] S. Fatikow, J. Seyfried, St. Fahlbuschand, A. Buerkle, and F. Schmoeckel, A flexible microrobot-based microassembly station, Journal of Intelligent and Robotic Systems, vol. 27, 2000, pp. 135169.

[5] E. Malis, Improving vision-based control using efficient second-order minimization techniques, Proceedings of IEEE International Conference on Robotics and Automation, New-Orleans, USA, May, 2004, pp. $1843-1848$.

[6] J. Minguez, F. Lamiraux, and J.P. Laumond, Obstacle avoidance techniques, Springer Handbook of Robotics, 2008, pp. 839-841.

[7] P. Song and V. Kumar, A potential field based approach to multirobot manipulation, IEEE Conference on Robotics and Automation, Washington, DC, USA, 2002, pp. 1217-1222.

[8] D. O. Popa and H. E. Stephanou, Micro and mesoscale robotic assembly, Journal of Manufacturing Process, vol. 6 (1), 2004, pp. $52-$ 71.

[9] S. Ralis, B. Vikramaditya, and B. J. Nelson, Micropositioning of a weakly calibrated microassembly system using coarse-to-fine visual servoing strategies, IEEE Transactions on Electronics Packaging Manufacturing, vol. 23 (2), 2000, pp. 123-131.

[10] G. Silveira and E. Malis, Real time tracking under arbitrary illumination changes, In Computer Vision and Pattern Recognition, Minneapolis, MIN, USA, 2007, pp. 1-6.

[11] W.T. Sun and T.C. Chin, Image-based visual servo for micromanipulation : a multiple-view and multiple-scale approach, International Symposium on Micro-Nanomechatronics and Human Science, 2004, pp. 136-141.

[12] B. Tamadazte, T. Arnould, S. Dembélé, N. Le Fort-Piat, and E. Marchand, Real-time vision-based microassembly of $3 D \mathrm{MEMS}$, IEEE/ASME International Conference on Advanced Intelligent Mechatronics, Singapore, July 2009, pp. 88-93.

[13] B. Tamadazte, S. Dembélé, G. Fortier, and N. Le Fort-Piat, Automatic micromanipulation using multiscale visual servoing, IEEE Conference on Automation Science and Engineering, Washington, USA, August 2008, pp. 977-982.

[14] W. Wong, X. Liu, and Y. Sun, Robust contact detection in micromanipulation using computer vision microscopy, In Proceeding IEEE Engineering Medical Biological Society, 2006, pp. 2219-2222.

[15] G. Yang, J. Gaines, and B. Nelson, A supervisory wafer-level 3D microassembly system for hybrid MEMS fabrication, Journal of Intelligent and Robotic Systems, vol. 37, 2003, pp. 43-68. 